Research Article

Robert V. Namm* and Georgiy I. Tsoy

\title{
Modified duality methods for solving an elastic crack problem with Coulomb friction on the crack faces
}

https://doi.org/10.1515/comp-2020-0147

Received Oct 29, 2019; accepted Apr 13, 2020

Abstract: We consider an equilibrium problem for an elastic body with a crack, on the faces of which unilateral nonpenetration conditions and Coulomb friction are realized. This problem can be formulated as quasi-variational inequality. To solve it, the successive approximation method is applied. On each outer step of this method, we solve an auxiliary problem with given friction. We solve the auxiliary problem by using modified Lagrange functionals. $\mathrm{Nu}-$ merical results are presented.

Keywords: elastic crack problem, duality scheme, Lagrange functional, Uzawa method, quasi-variational inequality

\section{Introduction}

The classical statement of an equilibrium problem for an elastic body with a crack is to specify equality-type boundary conditions on the crack faces. As a rule, such boundary conditions correspond to zero surface forces. However, these conditions do not exclude possible mutual penetration between the crack faces, which is unnatural from the standpoint of solid mechanics. There are numerical examples showing that such mutual penetration is possible [13]. Therefore, in recent papers on the crack theory, models with inequality-type boundary conditions on the crack faces are considered, providing mutual non-penetration between the crack faces. At the same time, problems with Coulomb friction on the crack faces are being investigated in solid mechanics. The simultaneous consideration of

*Corresponding Author: Robert V. Namm: Computing Center of the Far Eastern Branch of the Russian Academy of Sciences, Khabarovsk, Russia; Email: rnamm@yandex.ru

Georgiy I. Tsoy: Computing Center of the Far Eastern Branch of the Russian Academy of Sciences, Khabarovsk, Russia; Email: tsoy.dv@mail.ru the boundary conditions of non-penetration and Coulomb friction leads to quasi-variational inequalities the study of which is very delicate. To prove the existence of a solution to the quasi-variational inequality, fixed-point theorems are used $[4,5]$.

To solve the auxiliary problem with given friction, modified Lagrange functionals are used in the paper. The use of modified Lagrange functionals ensures the convergence of the Uzawa method with respect to the dual variable which is in contrast with the classical analogues.

The rest of this paper is structured as follows. Section 2 describes the initial-boundary value problem, the corresponding quasi-variational formulation and successive approximation method for solving the considering problem. In section 3 we introduce the duality method based on the modified Lagrange functional to solve the auxiliary problems. Section 4 presents the results of the numerical solution using the finite element method. Finally, Section 5 concludes the paper.

\section{Statement of the problem}

We investigate an equilibrium problem for an elastic body with a crack and assume that both the Coulomb friction law and a nonpenetration condition hold at the crack faces.

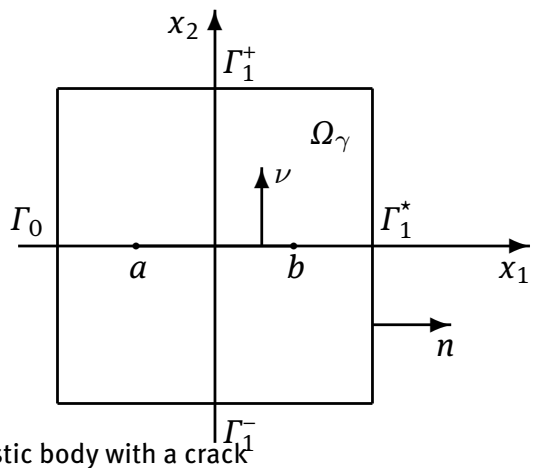

Figure 1: Elastic body with a crack

¿ Open Access. ( 2020 R. V. Namm and G. I. Tsoy, published by De Gruyter Attribution 4.0 License 
Let $\Omega \subset R^{2}$ be a bounded domain with a regular boundary $\Gamma$, and let $\gamma \subset \Omega$ be a cut(crack) inside $\Omega$. Assume that $\Gamma=\Gamma_{0} \bigcup \Gamma_{1}$, where $\Gamma_{0}, \Gamma_{1}$ are non-empty open disjoint subsets of $\Gamma$ and $\Gamma_{1}=\Gamma_{1}^{+} \bigcup \Gamma_{1}^{-} \bigcup \Gamma_{1}^{*}$ (see Figure 1).

Let $\gamma=\left\{\left(x_{1}, x_{2}\right) \in \Omega: a<x_{1}<b, x_{2}=0\right\}$, assuming that the end points $(a, 0)$ and $(b, 0)$ are crack vertices that do not reach the outer boundary $\Gamma$. Let $\Omega_{\gamma}=\Omega \backslash \bar{\gamma}, \bar{\gamma}=$ $\left\{\left(x_{1}, x_{2}\right) \in \Omega: a \leq x_{1} \leq b, x_{2}=0\right\}$. Let $\nu$ be the vector of the unit normal on $\gamma$. In this case on the crack $\gamma$ denote the positive (upper) face by $\gamma^{+}$and the negative (lower) face $\gamma^{-}$, where the signs \pm correspond to the positive and negative directions of the normal $\nu$ to $\gamma$. We consider the following equilibrium problem of two-dimensional elastic body with a crack and Coulomb friction between the crack faces.

For the displacement vector $v=\left(v_{1}, v_{2}\right)$, define the strain tensor

$$
\varepsilon_{i j}(v)=\frac{1}{2}\left(\frac{\partial v_{i}}{\partial x_{j}}+\frac{\partial v_{j}}{\partial x_{i}}\right), i, j=1,2,
$$

and the stress tensor

$$
\sigma_{i j}(v)=c_{i j k m} \varepsilon_{k m}(v),
$$

where $C=\left\{c_{i j k m}\right\}$ is a given elasticity tensor with the usual properties of positive definiteness and symmetry: $c_{i j k m}=$ $c_{j i m k}=c_{k m i j}, i, j, k, m=1,2, c_{i j k m} \in L^{\infty}(\Omega)$. Summation over repeated indices is assumed.

Let us specify vector-functions of the body and surface forces $f=\left(f_{1}, f_{2}\right)$ and $p=\left(p_{1}, p_{2}\right)$, respectively. The boundary-value problem is formulated as follows [5]:

$$
\begin{gathered}
-\frac{\partial \sigma_{i j}}{\partial x_{j}}=f_{i} \text { in } \Omega_{\gamma}, i=1,2, \\
u=0 \text { on } \Gamma_{0}, \\
\sigma_{i j} n_{j}=p_{i} \text { on } \Gamma_{1}, i=1,2,
\end{gathered}
$$

where $n=\left(n_{1}, n_{2}\right)$ is the unit outward normal vector to $\Gamma$.

The following conditions are set on $\gamma$ :

$$
\begin{gathered}
{\left[u_{\nu}\right] \geq 0,\left[\sigma_{\nu}(u)\right]=0, \sigma_{\nu}(u)\left[u_{\nu}\right]=0 \text { on } \gamma,} \\
\sigma_{\nu}(u) \leq 0, \text { on } \gamma^{ \pm} . \\
{\left[\sigma_{\tau}(u)\right]=0,\left|\sigma_{\tau}(u)\right| \leq-\mathcal{F} \sigma_{\nu}(u),} \\
\sigma_{\tau}(u)\left[u_{\tau}\right]+\mathcal{F} \sigma_{\nu}(u)\left|\left[u_{\tau}\right]\right|=0 \text { on } \gamma .
\end{gathered}
$$

Here $u_{\nu}=u \cdot \nu,\left[u_{\nu}\right]=u_{\nu}^{+}-u_{\nu}^{-}, \sigma_{\nu}(u)=\sigma_{i j}(u)_{\nu_{i}} \nu_{j},\left[\sigma_{\nu}(u)\right]=$ $\sigma_{\nu}^{+}(u)-\sigma_{\nu}^{-}(u), \sigma_{\tau}(u)=\sigma(u)-\sigma_{\nu} \nu,\left[\sigma_{\tau}(u)\right]=\sigma_{\tau}^{+}(u)-\sigma_{\tau}^{-}(u)$, where $\sigma(u)=\left(\sigma_{1}(u), \sigma_{2}(u)\right), \sigma_{i}(u)=\sigma_{i j}(u)_{\nu_{j}}, i=1,2$, $\mathcal{F}$ is the friction coefficient with $\mathcal{F} \geq 0$ on $\gamma$.

Define the set of admissible displacements

$$
K=\left\{v \in\left[H^{1}\left(\Omega_{\gamma}\right)\right]^{2}:\left[v_{\nu}\right] \geq 0 \text { on } \gamma, v=0 \text { on } \Gamma_{0}\right\},
$$

where, as before, $\left[v_{\nu}\right]=v_{\nu}^{+}-v_{\nu}^{-}$is a jump of the function $v_{\nu}=v \nu$ on $\gamma$. The values of the function $v_{\nu}$ on the crack $\gamma$ are thought of as values of the trace of the function $v_{\nu}$ on $\gamma, v_{\nu}^{ \pm} \in H^{1 / 2}(\gamma)[6]$

Let $f \in\left[L_{2}(\gamma)\right]^{2}$ and $p \in\left[L_{2}\left(\Gamma_{1}\right)\right]^{2}$. Assume that a solution $u \in K$ to the boundary-value problem (1), (2) exists and belongs to the class $\left[H^{2}\left(\Omega_{\gamma}\right)\right]^{2}$. Then $u$ satisfies the quasi-variational inequality $[5,7,8]$

$$
\begin{gathered}
a(u, v-u)+\int_{\gamma} \mathcal{F}\left|\sigma_{\nu}(u)\right|\left(\left|\left[v_{\tau}\right]\right|-\left|\left[u_{\tau}\right]\right|\right) d \Gamma \geq \\
\int_{\Omega_{\gamma}} f \cdot(v-u) d \Omega+\int_{\Gamma_{1}} p \cdot(v-u) d \Gamma \quad \forall v \in K,
\end{gathered}
$$

where

$$
a(u, v)=\int_{\Omega_{\gamma}} \sigma_{i j}(u) \varepsilon_{i j}(v) d \Omega=\int_{\Omega_{\gamma}} c_{i j p m} \varepsilon_{p m}(u) \varepsilon_{i j}(v) d \Omega
$$

is a bilinear form defined on $\left[H^{1}\left(\Omega_{\gamma}\right)\right]^{2}$.

Let us introduce the space $H_{00}^{1 / 2}(\gamma)$ :

$$
H_{00}^{1 / 2}(\gamma)=\left\{w \in H^{1 / 2}(\gamma): \frac{w}{\sqrt{\rho}} \in L_{2}(\gamma)\right\}
$$

with the norm

$$
\|w\|_{H_{00}^{1 / 2}(\gamma)}^{2}=\|w\|_{H^{1 / 2}(\gamma)}^{2}+\left\|\frac{w}{\sqrt{\rho}}\right\|_{L^{2}(\gamma)}^{2},
$$

where $\rho(x)=\operatorname{dist}(x, \partial \gamma)[6]$.

The quasi-variational inequality (3) can be formulated in the following way

$$
\begin{aligned}
u \in \arg \min _{v \in K}\{ & \left\{\frac{1}{2} a(v, v)-\int_{\Omega_{\gamma}} f \cdot v d \Omega-\int_{\Gamma_{1}} p \cdot v d \Gamma\right. \\
+ & \left.\int_{\gamma} \mathcal{F}\left|\sigma_{\nu}(u)\right|\left|\left[v_{\tau}\right]\right| d \Gamma\right\}
\end{aligned}
$$

The question of the existence of a solution to the quasivariational inequality reduces to the existence of a fixed point of mapping $T_{\gamma}:\left[H^{1}\left(\Omega_{\gamma}\right)\right]^{2} \rightarrow H_{00}^{-1 / 2}(\gamma)$ for small friction coefficients $\mathcal{F}$. Here, the element $T_{\gamma}(u)$ defines the functional that acts according to the rule

$$
\left(T_{\gamma}(u),[v]\right)=a(u, v)-\int_{\Omega_{\gamma}} f \cdot v d \Omega-\int_{\Gamma_{1}} p \cdot v d \Gamma
$$

for all $v \in\left[H^{1}\left(\Omega_{\gamma}\right)\right]^{2}$ such that $v=0$ on $\Gamma_{0}$ and $\left[v_{\tau}\right]=0$ on $\gamma[4,5]$.

The existence of a fixed point of the mapping $T_{\gamma}$ generates a heuristic method of successive approximations for solving a quasi-variational inequality [4, 9]:

1) Set an initial friction force $g^{0} \in H^{1 / 2}(\gamma)$. 
2) Find $u^{k}$ as a solution to the auxiliary variational inequality

$$
\begin{gathered}
a\left(u^{k}, v-u^{k}\right)+\int_{\gamma} g^{k}\left(\left|\left[v_{\tau}\right]\right|-\left|\left[u_{\tau}^{k}\right]\right|\right) d \Gamma \geq \\
\int_{\Omega_{\gamma}} f \cdot\left(v-u^{k}\right) d \Omega+\int_{\Gamma_{1}} p \cdot\left(v-u^{k}\right) d \Gamma \quad \forall v \in K .
\end{gathered}
$$

3) Calculate the approximation $g^{k+1}=\mathcal{F}\left|\sigma_{\nu}\left(u^{k}\right)\right|$.

It is easy to show that the variational inequality (5) is equivalent to the minimization problem

$$
\left\{\begin{array}{l}
J(v) \rightarrow \min \\
v \in K
\end{array}\right.
$$

Here $J(v)$ is the energy functional on the space $\left[H^{1}\left(\Omega_{\gamma}\right)\right]^{2}$ :

$$
J(v)=\frac{1}{2} a(v, v)-\int_{\Omega_{\gamma}} f \cdot v d \Omega-\int_{\Gamma_{1}} p \cdot v d \Gamma+\int_{\gamma} g^{k}\left|\left[v_{\tau}\right]\right| d \Gamma .
$$

It is known that there exists a unique solution $u \in K$ of the problem (6), which satisfies equilibrium equations and boundary conditions in a weak sense $[4,5]$.

To solve the problem (6) we describe a duality method based on the modified Lagrange functional that allows us simultaneously to drop the constraints $-\left[v_{\nu}\right] \leq 0$ on $\gamma$ and to smooth the minimizing functionals $J(v)$. [10]

Let us rewrite the problem (6) in an equivalent form

$$
\left\{\begin{array}{l}
\bar{J}(v, w) \rightarrow \min , \\
v \in K, w \in L_{2}(\gamma), w=0 \text { on } \gamma,
\end{array}\right.
$$

where the functional $\bar{J}(v, w)$ is defined as follows

$$
\begin{gathered}
\bar{J}(v, w)=\frac{1}{2} a(v, v)-\int_{\Omega_{\gamma}} f \cdot v d \Omega-\int_{\Gamma_{1}} p \cdot v d \Gamma+ \\
+\int_{\gamma} g^{k}\left|\left[v_{\tau}\right]-w\right| d \Gamma .
\end{gathered}
$$

Remark 1. In a subsequent section of this paper, we will investigate the problem (7) instead of the problem (6).

The non-differentiable functional of the problem (7) admits smoothing. The smoothing process is described in the next section.

\section{Modified duality scheme for solving the auxiliary problem}

Duality methods based on the classical Lagrange functional allow us to obtain convergence with respect to the primal variable only. To overcome this difficulty, we consider the modified duality scheme.

Let us introduce the modified Lagrange functional

$$
\begin{gathered}
M\left(v, w, l_{1}, l_{2}\right)=\bar{J}(v, w)+ \\
+\frac{1}{2 r} \int_{\gamma}\left(\left[\left(l_{1}-r\left[v_{\nu}\right]\right)^{+}\right]^{2}-l_{1}^{2}\right) d \Gamma+\int_{\gamma}\left(l_{2} w+\frac{r}{2} w^{2}\right) d \Gamma
\end{gathered}
$$

for $\left(v, w, l_{1}, l_{2}\right) \in\left[H_{\Gamma_{0}}^{1}\left(\Omega_{\gamma}\right)\right]^{2} \times\left[L_{2}(\gamma)\right]^{3}$, where $\left(l_{1}-\right.$ $\left.r\left[v_{\nu}\right]\right)^{+} \equiv \max \left\{0, l_{1}-r\left[v_{\nu}\right]\right\}$ and $r$ is an arbitrary positive constant.

Definition 1. A pair $\left(v^{\star}, w^{\star}, l_{1}^{\star}, l_{2}^{\star}\right) \in\left[H_{\Gamma_{0}}^{1}\left(\Omega_{\gamma}\right)\right]^{2} \times\left[L_{2}(\gamma)\right]^{3}$ is called a saddle point of the modified Lagrange functional $M\left(v, w, l_{1}, l_{2}\right)$ if the following two-sided inequality takes place:

$$
M\left(v^{\star}, w^{\star}, l_{1}, l_{2}\right) \leq M\left(v^{\star}, l^{\star}, l_{1}^{\star}, l_{2}^{\star}\right) \leq M\left(v, w, l_{1}^{\star}, l_{2}^{\star}\right)
$$

for $\forall\left(v, w, l_{1}, l_{2}\right) \in\left[H_{\Gamma_{0}}^{1}\left(\Omega_{\gamma}\right)\right]^{2} \times\left[L_{2}(\gamma)\right]^{3}$.

Let us consider the corresponding dual problem

$$
\left\{\begin{array}{l}
\underline{M}\left(l_{1}, l_{2}\right) \rightarrow \sup , \\
\left(l_{1}, l_{2}\right) \in\left[L_{2}(\gamma)\right]^{2},
\end{array}\right.
$$

where

$$
\underline{M}\left(l_{1}, l_{2}\right)=\inf _{(v, w) \in\left[H_{\Gamma_{0}}^{1}\left(\Omega_{\gamma}\right)\right]^{2} \times L_{2}(\gamma)}
$$

Let $\left(v^{\star}, w^{\star}, l_{1}^{\star}, l_{2}^{\star}\right)$ be a saddle point of the modified Lagrange functional $M\left(v, w, l_{1}, l_{2}\right)$. We assume that a solution $u$ of the problem (5) belongs to the space $\left[H^{2}\left(\Omega_{\gamma}\right)\right]^{2}$ and, moreover, meas $\left\{x \in \gamma: \sigma_{\nu}(u)<0\right\}>0$. Then the saddle point of $M\left(v, w, l_{1}, l_{2}\right)$ has the form $\left(u, 0 ;-\sigma_{\nu}(u), l_{2}^{*}\right)$, and the vector-function $\left(-\sigma_{\nu}(u), l_{2}^{\star}\right)$ is a solution of the dual problem (8) [10, 11].

Denote $K_{\mu_{1}, \mu_{2}}=\left\{(v, w) \in\left[H_{\Gamma_{0}}^{1}\left(\Omega_{\gamma}\right)\right]^{2} \times L_{2}(\gamma):-\left[v_{\nu}\right] \leq\right.$ $\mu_{1}, w=\mu_{2}$ on $\left.\gamma\right\} \forall\left(\mu_{1}, \mu_{2}\right) \in\left[L_{2}(\gamma)\right]^{2}$.

We define a sensitivity functional on the space $\left[L_{2}(\gamma)\right]^{2}$

$$
\chi\left(\mu_{1}, \mu_{2}\right)=\left\{\begin{array}{l}
\inf _{(v, w) \in K_{\mu_{1}, \mu_{2}}} \bar{J}(v, w), \text { if } K_{\mu_{1}, \mu_{2}} \neq \varnothing, \\
+\infty-\text { overwise . }
\end{array}\right.
$$

It can be shown that $\chi\left(\mu_{1}, \mu_{2}\right)$ is a proper convex functional in $\left[L_{2}(\gamma)\right]^{2}$, but its effective domain does not coincide with $\left[L_{2}(\gamma)\right]^{2}$. Obviously, $\overline{\operatorname{dom} \chi}=\left[L_{2}(\gamma)\right]^{2}$.

The dual functional has the other representation [12, 13]

$$
\begin{gathered}
\underline{M}\left(l_{1}, l_{2}\right)=\inf _{\left(\mu_{1}, \mu_{2}\right) \in\left[L_{2}(\gamma)\right]^{2}}\left\{\chi\left(\mu_{1}, \mu_{2}\right)+\right. \\
\left.+\int_{\gamma}\left(l_{1} \mu_{1}+l_{2} \mu_{2}\right) d \Gamma+\frac{r}{2} \int_{\gamma}\left(\mu_{1}^{2}+\mu_{2}^{2}\right) d \Gamma\right\} .
\end{gathered}
$$

Let us formulate two theorems for the sensitivity functional $\chi\left(\mu_{1}, \mu_{2}\right)[13]$. 
Theorem 1. Let $\left(\bar{\mu}_{1}, \bar{\mu}_{2}\right) \notin$ dom $\chi$. Then for any sequence $\left\{\left(\mu_{1}^{i}, \mu_{2}^{i}\right)\right\}$ in dom $\chi$, such that $\lim _{i \rightarrow \infty} \|\left(\mu_{1}^{i}, \mu_{2}^{i}\right)-$ $\left(\mu_{1}, \mu_{2}\right) \|_{\left[L_{2}(\gamma)\right]^{2}}=0$ the formula $\lim _{i \rightarrow \infty} \chi\left(\mu_{1}^{i}, \mu_{2}^{i}\right)=+\infty$ is correct.

Theorem 2. Let $\left\{\left(\mu_{1}^{i}, \mu_{2}^{i}\right)\right\} \in \operatorname{dom} \chi$ be a sequence such that $\lim _{i \rightarrow \infty}\left\|\left(\mu_{1}^{i}, \mu_{2}^{i}\right)-\left(\bar{\mu}_{1}, \bar{\mu}_{2}\right)\right\|_{\left[L_{2}(\gamma)\right]^{2}}=0$. Then $\varliminf_{i \rightarrow \infty} \chi\left(\mu_{1}^{i}, \mu_{2}^{i}\right) \geq \chi\left(\bar{\mu}_{1}, \bar{\mu}_{2}\right)$.

From theorems 1 and 2 it follows that the functional $\chi\left(\mu_{1}, \mu_{2}\right)$ is lower semicontinuous in $\left[L_{2}(\gamma)\right]^{2}$. Taking into account that $\chi\left(\mu_{1}, \mu_{2}\right)$ is a convex functional we can conclude that $\chi\left(\mu_{1}, \mu_{2}\right)$ is a weakly lower semicontinuous functional in $\left[L_{2}(\gamma)\right]^{2}$.

Since $\chi\left(\mu_{1}, \mu_{2}\right)$ is a weakly lower semicontinuous functional in $\left[L_{2}(\gamma)\right]^{2}$, we can consider the following Uzawa algorithm to determine the saddle point of the modified Lagrange functional [13]:

Step 0. $i:=0,\left(l_{1}^{0}, l_{2}^{0}\right) \in\left[L_{2}(\gamma)\right]^{2}$.

Step 1. Solve the problem:

$$
\left(v^{i+1}, w^{i+1}\right)=\underset{(v, w) \in\left[H_{\Gamma_{0}}^{1}\left(\Omega_{\gamma}\right)\right]^{2} \times L_{2}(\gamma)}{\arg \min } M\left(v, l_{2}^{i}\right) .
$$

Step 2. Set

$$
\begin{gathered}
\left(l_{1}^{i+1}, l_{2}^{i+1}\right)=\left(l_{1}^{i}, l_{2}^{i}\right)+ \\
+r\left(\max \left(-\left[v_{\nu}\right]^{i+1},-\frac{l_{1}^{i}}{r}\right), w^{i+1}\right) .
\end{gathered}
$$

Step 3. $i:=i+1$, back to Step 1 .

Remark 2. We solve problem (7) instead of problem (6). It allows us to obtain a minimization problem for the differentiable with respect to $v$ functional on Step 1 of the method.

In fact,

$$
\begin{gathered}
\min _{v, w} M\left(v, w, l_{1}, l_{2}\right)=\min _{v}\left(\frac{1}{2} a(v, v)-\int_{\Omega_{\gamma}} f \cdot v d \Omega-\right. \\
-\int_{\Gamma_{1}} p \cdot v d \Gamma+\frac{1}{2 r} \int_{\gamma}\left(\left[\left(l_{1}-r\left[v_{\nu}\right]\right)^{+}\right]^{2}-l_{1}^{2}\right) d \Gamma+ \\
\left.+\int_{\gamma} \inf _{w}\left(g^{k}|[v \cdot \tau]-w|+l_{2} w+\frac{r}{2} w^{2}\right) d \Gamma\right) .
\end{gathered}
$$

It is known that the number function

$$
F\left(v_{\tau}\right)=\inf _{w}\left(g^{k}|[v \cdot \tau]-w|+l_{2} w+\frac{r}{2} w^{2}\right)
$$

is a continuously differentiable convex function of real argument $(v \cdot \tau)[10]$.

Therefore, Step 1 of method (10) is reduced to the minimization problem of the differentiable with respect to $v$ functional

$$
\begin{gathered}
\frac{1}{2} a(v, v)-\int_{\Omega_{\gamma}} f \cdot v d \Omega-\int_{\Gamma_{1}} p \cdot v d \Gamma+ \\
+\frac{1}{2 r} \int_{\gamma}\left(\left[\left(l_{1}-r\left[v_{\nu}\right]\right)^{+}\right]^{2}-l_{1}^{2}\right) d \Gamma+\int_{\gamma} F(v \cdot \tau) d \Gamma .
\end{gathered}
$$

After finding $v^{i+1}$ we compute $w^{i+1}$ as follows

$$
w^{i+1}= \begin{cases}-\frac{g^{k}+l_{2}^{i}}{r}, & {\left[v_{\tau}^{i+1}\right]<-\frac{g^{k}+l_{2}^{i}}{r},} \\ {\left[v_{\tau}^{i+1}\right],} & -\frac{g^{k}+l_{2}^{i}}{r} \leq\left[v_{\tau}^{i+1}\right] \leq \frac{g^{k}-l_{2}^{i}}{r}, \\ \frac{g^{k}-l_{2}^{i}}{r}, & {\left[v_{\tau}^{i+1}\right]>\frac{g^{k}-l_{2}^{i}}{r} .}\end{cases}
$$

Thus, the proposed approach smooths the minimized functional of problem (7) and allows us to use effective methods of smooth optimization to solve it.

\section{Numerical experiment}

The domain $\Omega$ is taken to be a unit square with crack parameters $a=-0.3$ and $b=0.3$. For the numerical solution of the problem, we use the finite element method. We discretize the domain $\Omega_{\gamma}$ with a crack by a Delaunay triangulation and apply standard piecewise affine basis functions. Triangulation was performed with the use of Fade2D library 1.74 (www.geom.at). In the zones of maximum stress (near the crack), the mesh was thickened to improve the accuracy of the numerical solution. The domain $\Omega_{\gamma}$ was subdivided into 4290 triangles with $N=2329$ nodes. By $h=0.005$ we denote the corresponding mesh size on $\gamma$.

After a suitable discretization of (10) we have a vector $t=\left(t_{1}, \ldots, t_{2 N}\right)^{T} \in \mathbb{R}^{2 N}$ of unknowns, assembling the components of the displacement vector $\left(u_{1}\left(x^{m}\right), u_{2}\left(x^{m}\right)\right)^{T}$ in an appropriate way at the grid points $x^{m}$ with $m=\overline{1, N}$. Then the finite-dimensional minimization problem is reduced to finding the optimal values of $t_{i}$. For this, we use the generalized Newton method $[14,15]$.

Let us present the results of numerically solving the problem. The parameter values are as follows: $f=$ $\left(f_{1}, f_{2}\right)=(0,0)$, the surface force on the right side $\left.p\right|_{\Gamma_{1}^{*}}=$ $\left(27 \cdot \rho\left(x_{2}\right), 0\right) \mathrm{MPa}$, on the upper side $\left.p\right|_{\Gamma_{1}^{+}}=(0,-1) \mathrm{MPa}$ and on the lower side $\left.p\right|_{\Gamma_{1}^{-}}=(0,1) \mathrm{MPa}$, Young elasticity modulus $E=73000 \mathrm{MPa}$, Poisson coefficient $\mu=0.34$, constant $r=10^{8}$. The function $\rho\left(x_{2}\right)$ determines the distribution of the acting surface forces. Let $\rho\left(x_{2}\right)=\rho_{1}\left(x_{2}\right)$, 
where

$$
\rho_{1}\left(x_{2}\right)=\left\{\begin{array}{l}
1-\left|4 x_{2}-1\right|, \text { if } x_{2} \geq 0, \\
\left|4 x_{2}+1\right|-1, \text { if } x_{2} \leq 0 .
\end{array}\right.
$$

Numerical experiments were conducted on a hybrid computing cluster based on the OpenPOWER architecture. All cluster nodes are IBM Power Systems S822LC 8335-GTB servers with the following configuration:

- two IBM POWER8 processors with the maximum frequency of $4.023 \mathrm{GHz}$,

- two NVIDIA Tesla P100 coprocessors with 16 GB HBM2 memory,

- 256GB of 2400MHz DDR4 system memory,

- two Seagate 1 TB 7200 RPM hard disk drives,

- EDR InfiniBand controller.

Each processor IBM POWER8 contains 10 computing cores that support simultaneous multithreading (SMT) for 8 threads.

The main computational complexity of the generalized Newton method consists in finding the inverse generalized Hessian matrix. Therefore, the calculations were performed on NVIDIA Tesla P100 GPU using the cuBLAS library. To work with the coprocessors, we used NVIDIA CUDA Toolkit 10.1.168. Matrix inversion was carried out using functions cublasDgetrfBatched, cublasDgetriBatched for the matrix size $4658 \times 4658$.

The accuracy of the calculations for the primal variable is $\varepsilon_{t}=10^{-12}$, for the dual variable $\varepsilon_{l}=10^{-8}$.

Table 1 presents the results of the numerical solution using the modified Lagrange functional and shows the number of iterations for primal and dual variables on each step of the successive approximations method.

Table 1: Numerical results.

\begin{tabular}{cccccc}
\hline $\mathrm{k}$ & $\begin{array}{c}\text { Iter. by } \\
t\end{array}$ & $\begin{array}{c}\text { Iter. by } \\
\left(l_{1}, l_{2}\right)\end{array}$ & $\left\|l_{1}\right\|_{2}$ & $\left\|l_{2}\right\|_{2}$ & $\left\|g^{k}\right\|_{2}$ \\
\hline 1 & 3 & 8 & 10.000232 & 0 & 3.0000694 \\
2 & 4 & 8 & 9.9963171 & 3.0000694 & 2.9988946 \\
3 & 4 & 8 & 9.9963172 & 2.9988946 & 2.9988952 \\
4 & 4 & 8 & 9.9963173 & 2.9988956 & 2.9988956 \\
5 & 4 & 8 & 9.9963173 & 2.9988956 & 2.9988956 \\
\hline
\end{tabular}

The numerical calculations show fast convergence of the Uzawa algorithm with modified Lagrange functional.

The graphs on Figure 2 show the jump of the functions $\left[u_{\nu}\right]=0$ and $\left[u_{\tau}\right] \geq 0$ on the crack, so there is no mutual penetration between the crack faces into each other.

Crack faces are stuck together $\left(\left[u_{\nu}\right]=0\right)$, so it can be seen from Figure 3 that the value of the dual variable $l_{1}$ is

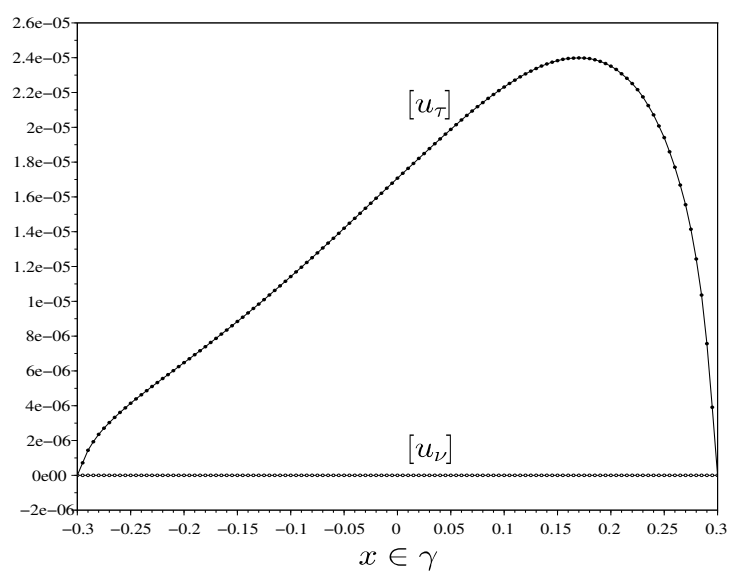

Figure 2: Jump of the functions $u_{\nu}, u_{\tau}$ on the crack with $\rho_{1}\left(x_{2}\right)$.

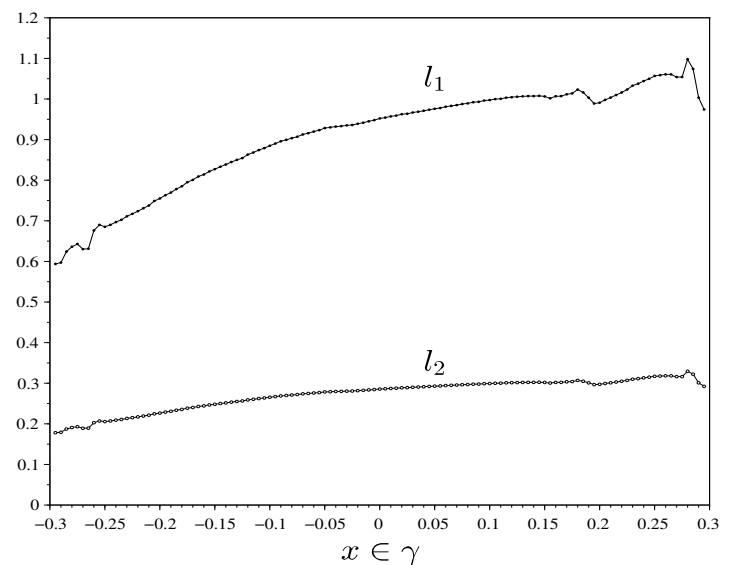

Figure 3: Value of the dual variables $l_{1}, l_{2}$ with $\rho_{2}\left(x_{2}\right)$.

greater than zero at all contact points. This indicates the presence of normal stress in these points.

Figures 4, 5 show the numerical solution of the problem with $\rho\left(x_{2}\right)=\rho_{2}\left(x_{2}\right)=1-\left|2 x_{2}\right|$. Such loading provides the closing of the crack faces in the vicinity of its left tip and an opening mode in the vicinity of the right one. As in the previous example, there is no mutual penetration between the crack faces and the dual variable $l_{1}$ is positive at the points where crack faces are stuck together.

Thus, the numerical calculations confirm that modified Lagrange functionals make it possible to efficiently solve elastic crack problems with the conditions of mutual non-penetration and Coulomb friction on the crack faces. 


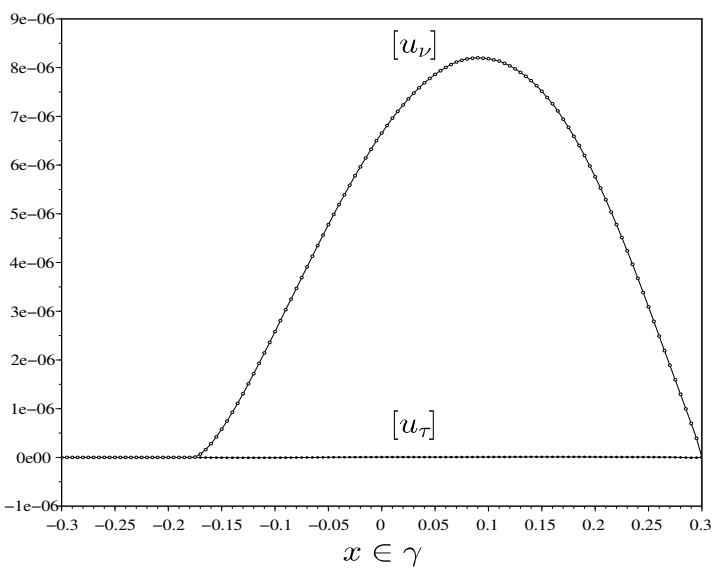

Figure 4: Jump of the functions $u_{\nu}, u_{\tau}$ on the crack with $\rho_{2}\left(x_{2}\right)$.

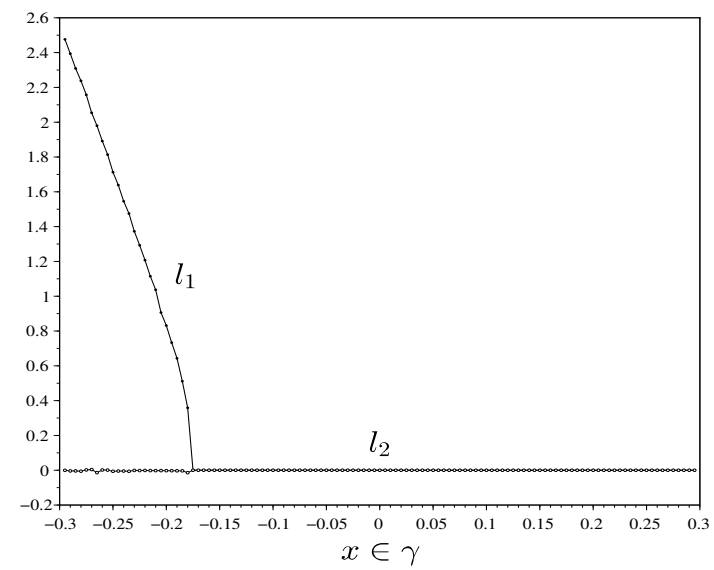

Figure 5: Value of the dual variables $l_{1}, l_{2}$ with $\rho_{2}\left(x_{2}\right)$.

\section{Conclusion}

In this paper, the authors investigated and numerically solved the quasi-variational inequality corresponding to the elastic crack problem with the conditions of mutual non-penetration and Coulomb friction. To solve auxiliary problems with given friction, we constructed the duality method based on the modified Lagrange functional. The suggested method allows us simultaneously to drop inequality-type constraints and to smooth the minimizing functionals. Moreover, the solution of the dual problem defines the friction force on the next step of the successive approximation method.

Modified Lagrange functionals make it possible to use the gradient method for solving the dual problem, which is more efficient in comparison with the gradient projection method used in the classical duality scheme. The numerical experiments confirm the fast convergence of the Uzawa algorithm with respect to the dual variable. At each step of the successive approximation method, only eight iterations of the Uzawa method were required. The numerical stabilization of solutions to auxiliary problems occurs very quickly. In the above example, five steps were required.

Acknowledgement: This study was supported by the Russian Foundation for Basic Research (Project 20-01-00450 A). Numerical experiments were performed on a computational cluster provided by the Shared Services Center “Data Center of FEB RAS” (Khabarovsk) [16].

\section{References}

[1] Hintermüller M., Kovtunenko V. A., Kunisch K., The primal-dual active set method for a crack problem with non-penetration, IMA Journal of Applied Mathematics, 2004, 69(1), 1-26

[2] Kovtunenko V.A., Numerical simulation of the nonlinear crack problem with nonpenetration, Mathematical Methods in the Applied Sciences, 2004, 27(2), 163-179

[3] Rudoy E.M., Domain decomposition method for crack problems with nonpenetration condition, ESAIM: Mathematical Modelling and Numerical Analysis, 2016, 50(4), 995-1009

[4] Glavaček I., Haslinger J., Nečas I., Lovišek J., Numerical Solution of Variational Inequalities, Springer-Verlag, Berlin, 1988

[5] Kovtunenko V.A., Crack in a solid under Coulomb friction law, Applications of Mathematics, 2000, 45(4), 265-290

[6] Khludnev A.M., Elastisy Problems in Nonsmooth Domains, Fizmatlit, Moscow, 2010 (in Russian)

[7] Kikuchi N., Oden T., Contact Problem in Elasticity: A Study of Variational Inequalities and Finite Element Methods, SIAM, Philadelphia, 1988

[8] Kravchuk A.S., Variational and Quasi-Variational Inequalities in Mechanics, MGAPI, Moscow, 1997 (in Russian)

[9] Namm R.V., Tsoy G.I., A method of successive approximations for solving the quasi-variational Signorini inequality, Russian Mathematics, 2017, 61(1), 39-46

[10] Kushniruk N.N., Namm R.V., Finite-element solution of a model mechanical problem with friction based on a smoothing Lagrange multiplier method, Computational Mathematics and Mathematical Physics, 2012, 52(1), 20-30

[11] Vikhtenko E.M., Namm R.V., Duality scheme for solving the semicoercive Signorini problem with friction, Computational Mathematics and Mathematical Physics, 2007, 47(12), 19381951

[12] Namm R.V., Vikhtenko E.M., Maksimova N.N., Sensitivity functionals in variational inequalities of mechanics and their applications to duality schemes, Numerical Analysis and Applications, 2014, 7(1), 36-44

[13] Namm R.V., Tsoy G.l., A modified dual scheme for solving an elastic crack problem, Numerical Analysis and Applications, 2017, 10(1), 37-46

[14] Mangasarian O.L., A generalized Newton method for absolute value equations, Optimization Letters, 2009, 3(1), 101-108 
[15] Namm R.V., Tsoy G.I., Vikhtenko E.M., A Modified Duality Method for Solving an Elasticity Problem with a Crack Extending to the Outer Boundary, Communications in Computer and Information Science, 2019, 974, 35--48
[16] Sorokin A.A., Makogonov S.V., Korolev S.P., The information infrastructure for collective scientific work in the far east of Russia, Scientific and Technical Information Processing, 2017, 44(4), 302-304 